\title{
Die Semitendinosus- und Gracilissehne als autologes Transplantat zur Sehnen- und Bandrekonstruktion
}

\author{
Wolf Petersen, Ute Petersen, Cara Winter
}

\section{Zusammenfassung}

Die Ansatzsehnen des M. semitendinosus und des $M$. gracilis gehören funktionell zu den Beugern des Kniegelenks und sie bilden zusammen mit dem M. sartorius den Pes anserinus superficialis. In der Kreuzbandchirurgie haben sich diese Sehnen als autologe Transplantate etabliert. Sie eignen sich aber auch als Transplantate für den Ersatz großer Sehnen wie z. B. der Achillessehne und der Patellarsehne. Auch der Ersatz der Peroneussehne, der Ersatz der Syndesmose oder der fibulotalaren Bänder sind mit diesen Transplantaten möglich. Die Indikation zum Sehneninterponat besteht bei längerstreckigen Defekten, die durch Verschiebetechniken oder Z-Plastiken nicht mehr rekonstruierbar sind.

\section{Einleitung}

Läsionen von Sehnen können zu erheblichen funktionellen Defiziten an der unteren Extremität führen. $\mathrm{Zu}$ den am häufigsten verletzten Sehnen zählt die Achillessehne. Aber auch Rupturen der Patellarsehne, Quadrizepssehne oder der Peronealsehnen kommen vor. Werden diese Verletzungen übersehen, können Defekte entstehen, die eine Rekonstruktion mit einem autologen Sehnentransplantat erforderlich machen. In der Kreuzbandchirurgie haben sich als autologe Sehnentransplantate die Semitendinosus- und Gracilissehne etabliert [6,7]. Diese Sehnen eignen sich auch als Transplantate für den Ersatz großer Sehnen wie z.B. der Achillessehne und der Patellarsehne $[2,5,9]$. Auch der Ersatz der Pe-

OP-JOURNAL 2015; 31: 182-187

(C) Georg Thieme Verlag KG Stuttgart · New York DOI http://dx.doi.org/10.1055/s-0041-109446

\section{The Semitendinosus and Gracilis Tendons as Autologous Tendon Grafts for Tendon and Ligament Reconstruction}

The tendons of the semitendinosus and gracilis muscles are flexors of the knee. Along with the sartorius muscle, they make up the superficial pes anserinus. In cruciate ligament surgery, these tendons are well established autologous grafts. They can also be used as a tendon graft for the reconstruction of large tendons such as the Achilles or patellar tendon, for reconstruction of the peroneus brevis tendon, replacement of the distal syndesmosis and reconstruction of the talofibular ligaments. The indication for a tendon interposition graft is given in case of a large defect when reconstruction with a sliding flap or a Z-plasty is not possible. wird die Sartoriusfaszie längs gespalten $[6,7]$.

Nach Spaltung der Sartoriusfaszie werden die Ansatzsehnen der Mm. semitendinosus und gracilis sichtbar. Die kaudal gelegene Ansatzsehne des M. semitendinosus wird mit einer Overholt-Klemme unterfahren und mit einer Fadenschlaufe umschlungen. Danach werden die Verbindungen der Semitendinosussehne zum M. gastrocnemius getrennt. Dabei wird die Sehne an der Fadenschlaufe gehalten. Anschließend wird die Sehne mithilfe der Fadenschlaufe in ein rundes Sehnenmesser („Sehnenstripper“, z.B. Karl Storz, Tuttlingen) geführt und die Sehne durch langsames Vorschieben parallel zur Oberschenkelachse entnommen. Dabei wird die Sehne mit einer feuchten Kompresse gehalten.

Die Ansatzsehne des M. gracilis kann auf gleiche Weise entnommen werden. Welche der beiden Sehnen entnommen wird, richtet sich nach der Größe des zu rekonstruierenden Defekts. Die Länge der Semitendinosussehne variiert zwischen 26 und $34 \mathrm{~cm}$, die der Gracilissehne zwischen 22 und $28 \mathrm{~cm}$.

Ziel dieses Beitrags ist es, die Entnahmetechnik der Semitendinosussehne und Gracilissehne sowie deren Einsatz als Transplantat zum Ersatz großer Sehnen oder Bänder zu beschreiben.

\section{Entnahme der Semitendinosus- oder der Gracilissehne}

Die Ansatzsehnen des M. semitendinosus und des M. gracilis gehören funktionell zu den Beugern des Kniegelenks und sie bilden zusammen mit dem M. sartorius den Pes anserinus superficialis. Sie inserieren ca. $5 \mathrm{~cm}$ unterhalb des Gelenkspalts medial der Tuberositas tibiae. Zur Sehnenentnahme wird ein ca. $3 \mathrm{~cm}$ langer Hautschnitt medial der Tuberositas tibiae angelegt (Abb. 1 und 2). Danach
Um die Sehne durch das Restgewebe der zu rekonstruierenden Sehne führen zu können, müssen die Sehnenenden mit resorbierbarem Fadenmaterial armiert werden.

\section{Ersatz der Achillessehne}

Die Achillessehne ist die kräftigste Sehne des menschlichen Körpers. Sie setzt sich zusammen aus den Ansatzsehnen der Mm. gastrocnemii und der Ansatzsehne des M. soleus. Die einzelnen Anteile der Achillessehne verlaufen jedoch nicht parallel. Die Ansatzsehne des M. gastrocnemius medialis inseriert im lateralen Anteil der Insertionszone am Kalkaneus, die Ansatzsehne des M. gastrocnemius lateralis inseriert medial und die Ansatzsehne des M. soleus inseriert im poste- 


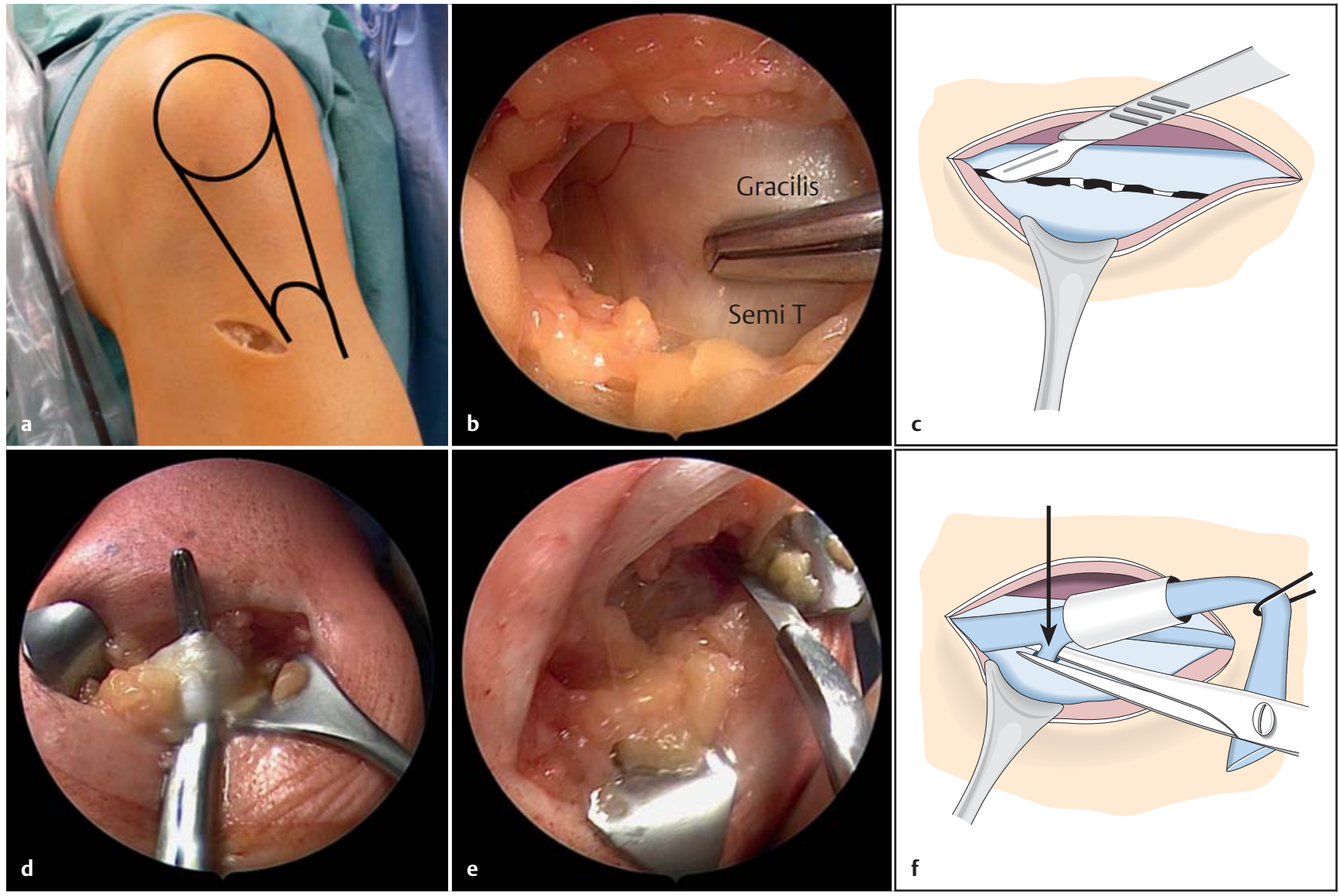

Abb. 1 a bis $\mathbf{f}$ Entnahme der Semitendinosussehne. a Der $3 \mathrm{~cm}$ lange Hautschnitt befindet sich medial der Tuberositas tibiae. $\mathbf{b}$ Die Sehnen können unter der Sartoriusfaszie palpiert werden. Die obere Sehne ist die Gracilissehne. Die untere Sehne ist die Semitendinosussehne. c Mit dem Skalpell wird die Sartoriusfaszie zwischen den Sehnen gespalten. d Die Semitendinosussehne wird mit der Overholt-Klemme unterfahren und mit einem Faden (Stärke 1) angeschlungen. e Mit einem Skalpell wird die Sehne an der Insertion abgelöst. f Mit einer Präparierschere werden die Verbindungen der Semitendinosussehne zum Gastrocnemiuskopf getrennt.

rioren Anteil. Auf diese Weise entsteht eine Torquierung der einzelnen Sehnenanteile.

Zur Rekonstruktion der Achillessehne erfolgt ein ca. 15-20 cm langer Hautschnitt medial der Achillessehne. Die mediale Schnittführung ist zur Schonung des $\mathrm{N}$. suralis und zur Vermeidung von Wundheilungskomplikationen erforderlich.

Danach wird das Paratenon eröffnet, um das restliche Sehnengewebe darzustellen. Die Sehnenstümpfe werden débridiert und es wird überprüft, ob eine Rekonstruktion durch eine lokale Verschiebeplastik erfolgen kann (z. B. VY-Plastik).

Bei Defektlängen von mehr als $5 \mathrm{~cm}$ wird meist ein autologes Sehnentransplantat benötigt. Infrage kommen die Ansatzsehne des M. flexor hallucis longus oder des M. peroneus brevis. Nachteil der Peroneus-brevis-Sehne ist das Risiko funktioneller OSG-Instabilitäten (OSG: oberes Sprunggelenk). Die Flexor-hallucis-
Sehne kann sehr dünn ausfallen. Daher sind die Semitendinosussehne oder die Gracilissehne eine gute Alternative als Sehnentranplantat [2].

Das Sehnentransplantat wird mit einer Overholt-Klemme quer durch den distalen Stumpf gezogen. Danach wird der Fuß in Plantarflexion gebracht und das Sehnentransplantat mit dem proximalen Stumpf vernäht (PDS, Stärke 3-0.). Das Sehnentransplantat kann nach der Fixation noch durch eine PDS-Kordel (Stärke $0,7 \mathrm{~mm}$ ) gesichert werden (Abb. 3). Ist distal kein ausreichender Stumpf mehr vorhanden, kann die Sehne auch durch einen Bohrkanal im Kalkaneus gezogen werden.

Danach wird das Paratenon genäht (PDS, Stärke 3-0), die Haut spannungsfrei verschlossen und der Patient mit einem Kompressionsverband versorgt. Noch im OP-Saal wird dem Patienten eine anteriore Gipsschale in Spitzfußstellung angelegt.

\section{Postoperative Behandlung}

Für 2 Wochen wird der Patient bis zur Wundheilung mit der anterioren Gipsschale behandelt. In dieser Zeit steht die Wundheilung im Vordergrund: Hochlagerung und Lymphdrainage. Danach wird dem Patienten eine Orthese, die den Fuß in Spitzfußstellung hält, angepasst (z.B. Vacoped). Die Spitzfußstellung wird alle 2 Wochen reduziert $\left(30^{\circ}\right.$, $\left.15^{\circ}, 0^{\circ}\right)$. In dieser Zeit wird mit $20 \mathrm{~kg}$ Teilkörpergewicht belastet.

\section{Ersatz der Patellarsehne}

Die Patellarsehne wird im Schrifttum auch als Lig. patellae bezeichnet, da sie die Verbindung zwischen Kniescheibe und Tuberositas tibiae ist. Funktionell ist sie aber als Sehne zu sehen, da die Patella als Sesambein in den Streckapparat eingelagert ist. Daher wird die Streckung durch eine Sehnenruptur erheblich beeinträchtigt. 

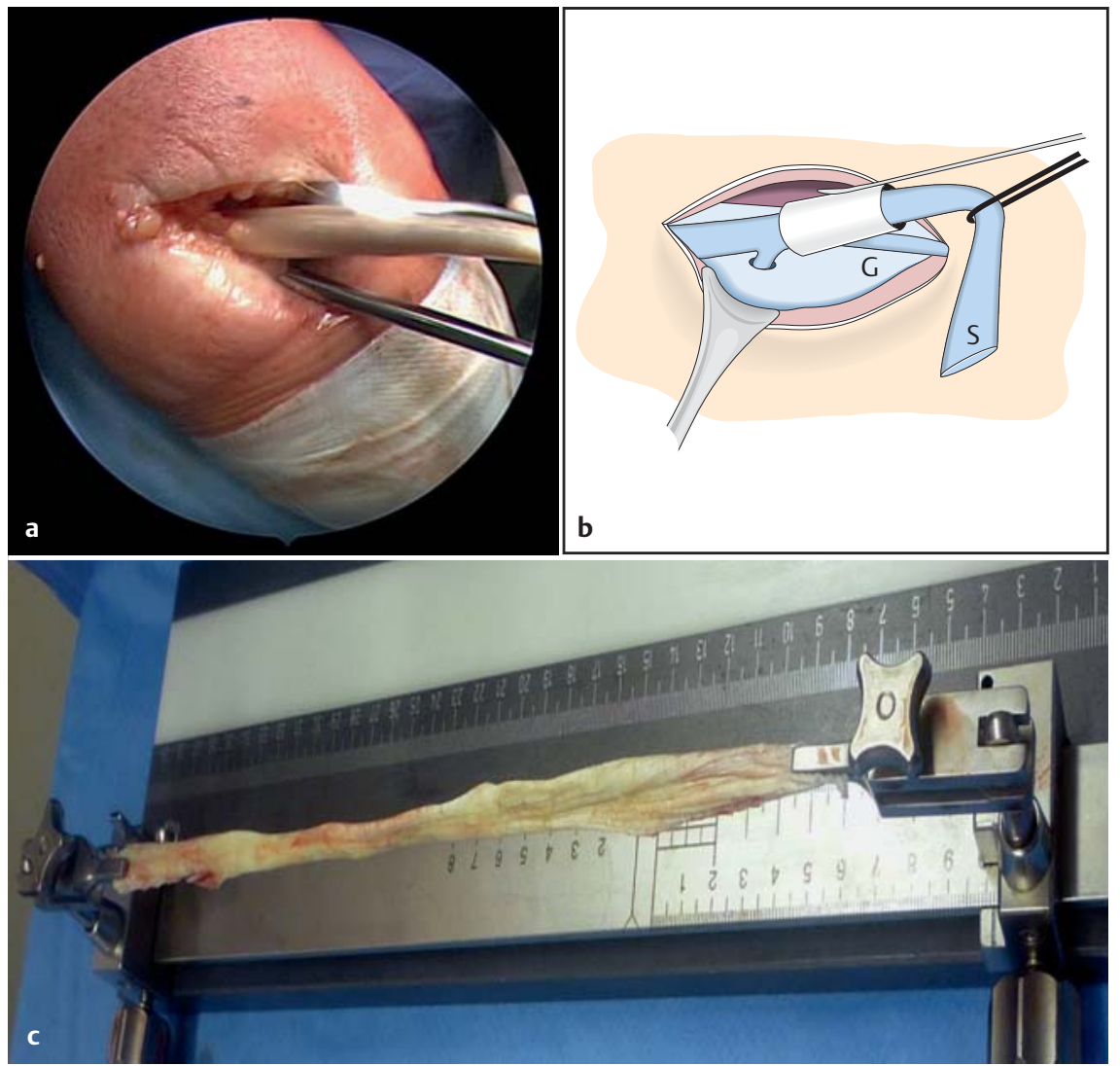

Abb. 2 a bis c Entnahme der Semitendinosussehne. a Mit einem Sehnenstripper wird die Semitendinosussehne entnommen. b Der Sehnenstripper muss parallel zum Femur geführt werden. c Auf einem speziellen Präparationsbrett kann die Sehne präpariert werden.

Als Schädigungsmechanismus für die Spontanruptur wird meist eine forcierte exzentrische Bewegung (z.B. beim Treppabgehen) angegeben. Beim alten Patienten ist eine Schädigung durch ein Hochrasanztrauma mit Knieluxation selten, aber durchaus möglich.

\section{Therapie}

Die Therapie der Patellarsehnenruptur ist die primäre offene Sehnenrekonstruktion. Diese sollte zeitnah innerhalb der ersten 1-2 Wochen nach Verletzung erfolgen.

\section{Operationstechnik bei veralteter Ruptur mit Defekt}

Der Zugang und die Darstellung der Sehne erfolgt ebenfalls über einen anterioren Längsschnitt. Liegt ein Sehnendefekt vor, kann die Sehne mit einem autologen Sehnentransplantat rekonstruiert werden $[5,9]$. Als Sehnentransplantat eignet sich die Ansatzsehne des M. semitendinosus (Abb. 4).

Zunächst sollte die korrekte Patellahöhe mit einer Rahmen-Cerclage eingestellt

\section{Postoperative Behandlung}

Obwohl die Draht-Cerclage intraoperativ einen stabilen Eindruck vermittelt, empfehlen wir eine 6-wöchige Teilbelastung. Je nach Zuverlässigkeit oder motorischen Fähigkeiten wird der Patient entweder für 6 Wochen mit einem Tutor oder mit einer beweglichen Knieorthese (0-0-30 2 Wochen, 0-0-60 weitere 2 Wochen und 0-0-90 weitere 2 Wochen) versorgt. In dieser Zeit wird mit $20 \mathrm{~kg}$ Teilkörpergewicht belastet. Aus dem Tutor heraus können passive Bewegungsübungen auf einer CPM-Schiene erfolgen. Die DrahtCerclage sollte nach 8-10 Wochen entfernt werden.

\section{Ersatz der Peroneus-brevis-Sehne}

Die Peronealsehnen unterstützen die Plantarflexion und sie wirken als funktionelle Stabilisatoren des oberen Sprunggelenks. Das bedeutet umgekehrt, dass chronische Instabilitäten des OSG zu Überlastungsschäden dieser Sehnen führen können. Liegt eine Kombination aus Instabilität und Peronealsehnentendinose vor, sollte immer auch die OSG-Instabilität therapiert werden.

Die Schädigung dieser Sehnen erfolgt nahezu immer ohne akutes Trauma als chronischer Überlastungsschaden. Reine Tendinosen werden zunächst konservativ behandelt. Bei Längsrissen kommt auch eine operative Intervention mit Sehnennaht in Betracht. Bei langstreckigen Defekten der Peronealsehnen kann der Ersatz der Peroneus-brevis-Sehne mit einem Gracilissehneninterponant indiziert sein [8]. Symptome bei Defekten der Peronealsehnen können schmerzhafte motorische Defizite sein (Beeinträchtigung der Fußaußenrandhebung).

\section{OP-Technik}

Die Exposition der Peroneus-brevis-Sehne erfolgt über eine bogenförmige Inzision, die von der Außenköchelhinterkante bis zur MT-5-Basis (MT: Metatarsale) reicht (Abb. 5). Danach wird die Sehnenscheide komplett geöffnet und der Defekt dargestellt. Wenn der Defekt für eine lokale Verschiebeplastik zu groß ist, kann eine Rekonstruktion mit einem Sehneninterponat indiziert sein. Als Interponat eignet sich die Gracilissehne.

Zunächst erfolgt eine Seit-zu-Seit-Naht mit dem proximalen Stumpf der Peroneus-brevis-Sehne. Distal erfolgt entweder eine Seit-zu-Seit-Naht mit dem dis- 

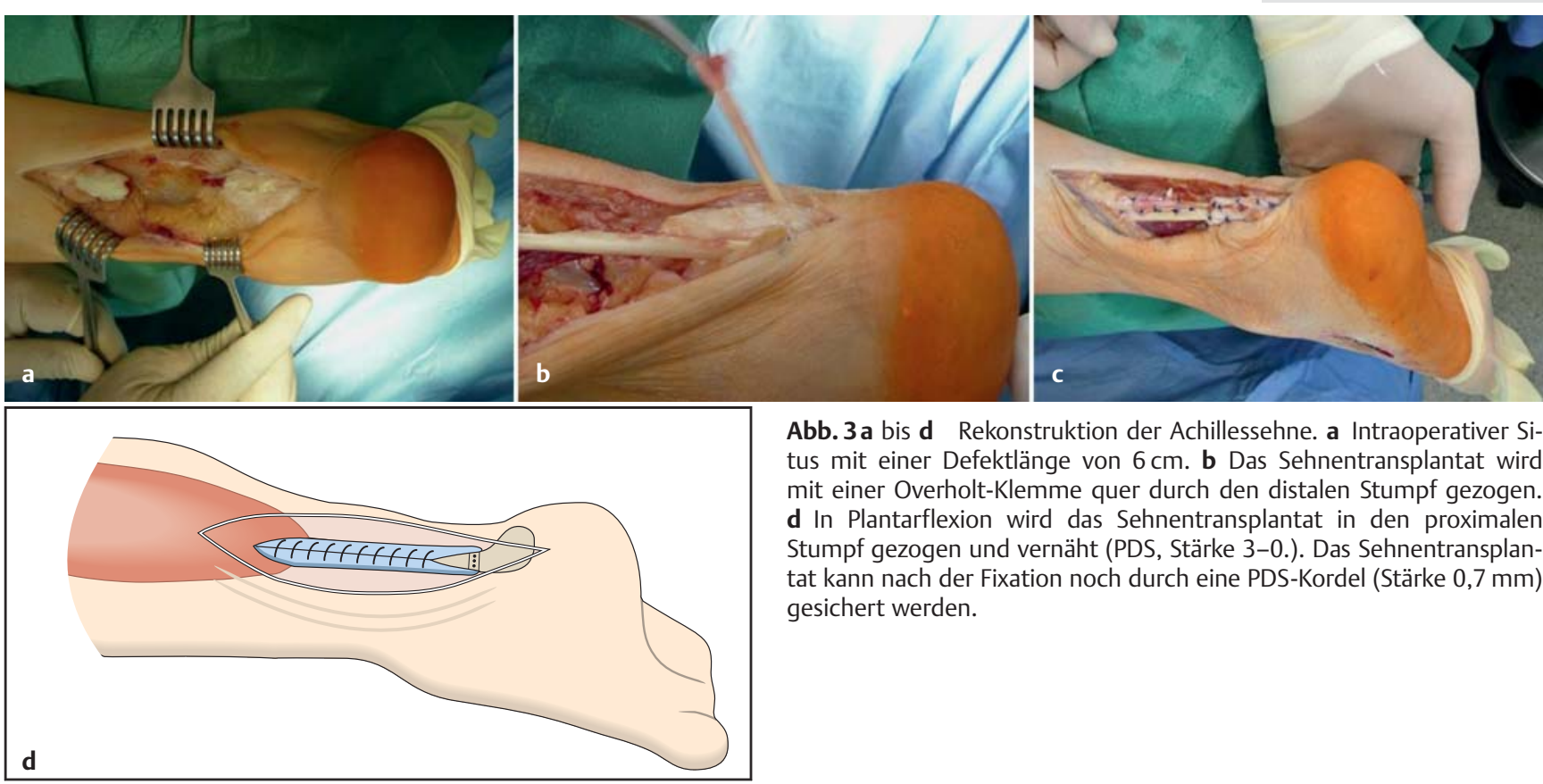

Abb. $\mathbf{3}$ a bis $\mathbf{d}$ Rekonstruktion der Achillessehne. a Intraoperativer Situs mit einer Defektlänge von $6 \mathrm{~cm}$. b Das Sehnentransplantat wird mit einer Overholt-Klemme quer durch den distalen Stumpf gezogen. d In Plantarflexion wird das Sehnentransplantat in den proximalen Stumpf gezogen und vernäht (PDS, Stärke 3-0.). Das Sehnentransplantat kann nach der Fixation noch durch eine PDS-Kordel (Stärke 0,7 mm) gesichert werden.

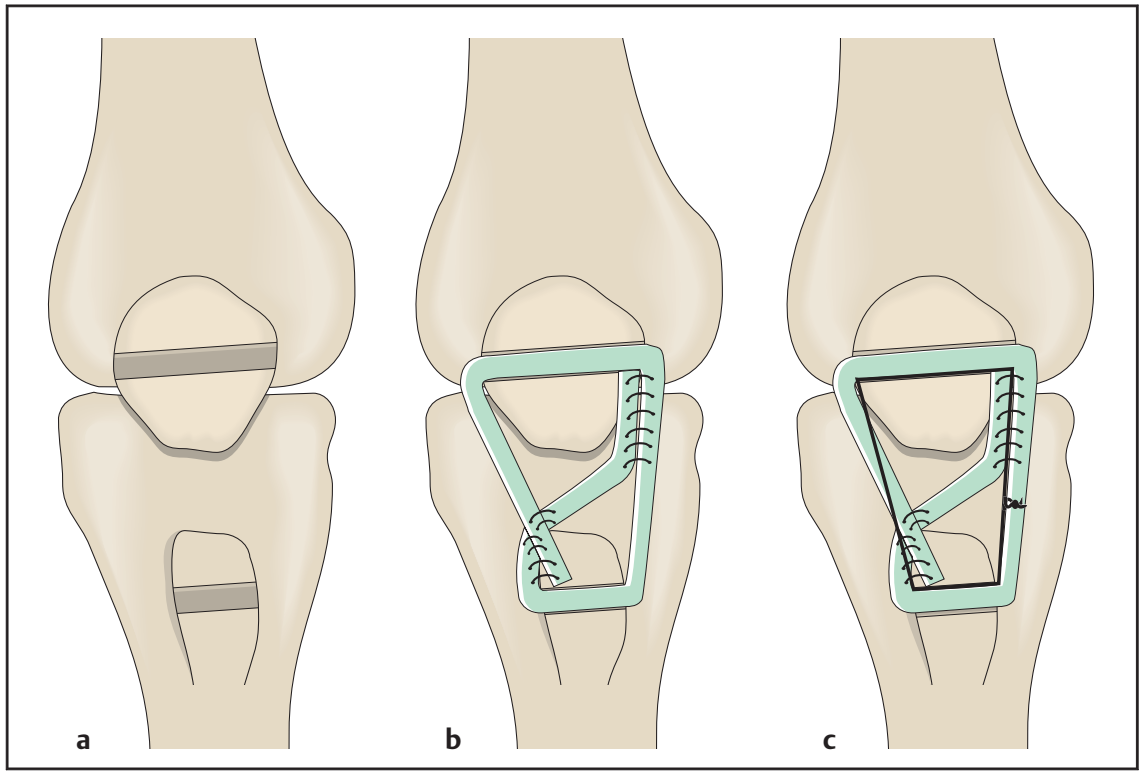

Abb. 4a bis c Rekonstruktion der Patellarsehne. a Transversale Bohrlöcher in der Tuberositas tibiae und im distalen Drittel der Patella (Durchmesser 4,5-5 mm). b Das Sehnentransplantat wird sowohl durch das transversale Bohrloch in der Tuberositas tibiae als auch das der Patella gezogen. Danach werden die beiden Sehnentransplantate miteinander vernäht, nachdem die richtige Höhe der Patella eingestellt wurde. c Durch eine Cerclage (Draht, PDS oder FiberwireKordel) wird das Sehnentransplantat gesichert.

talen Stumpf oder der Einzug in ein Bohrloch in der MT-5-Basis. Die Fixation im Knochentunnel kann entweder mit einer kleinen Interferenzschraube oder mit einem extrakortikalen Kippanker erfolgen. Die Reste der Peroneus-longusSehne können auf das Interponat aufgenäht werden.

\section{Postoperative Behandlung}

Postoperativ wird der Fuß in einer festen OSG-Orthese (z.B. Walker, Vacoped) für 8 Wochen immobilisiert.

In dieser Zeit wird mit 20 kg Teilkörpergewicht belastet.

\section{Ersatzplastik der Außenbänder bei chronischer OSG-Instabilität}

Chronische Instabilitäten des OSG können zu deutlichen funktionellen Beeinträchtigungen führen. Bessern sich die Instabilitäten durch neuromuskuläres Training nicht, kann eine Bandplastik indiziert sein.

Wenn noch Bandreste vorhanden sind, kann eine Raffung des Lig. fibulotalare anterius (LFTA) und fibulocalcaneare (LFC) sinnvoll sein (Broström-OP). Wenn kein Bandmaterial mehr vorhanden ist, sollten die Bänder durch ein Sehnentransplantat ersetzt werden [1]. Dabei werden anatomische Techniken von nicht anatomischen Techniken unterschieden. Nicht anatomische Techniken unter Verwendung der Peroneus-brevisSehne haben den Nachteil, dass diese Sehnen funktionelle Stabilisatoren des OSG sind. Als Transplantat für den anatomischen Bandersatz kommen die Semitendinosus- oder die Gracilissehne in Betracht.

\section{OP-Technik}

Die Exposition des lateralen Bandapparats erfolgt über eine bogenförmige Inzision entlang der Außenknöchelvorderkante, die ca. $6 \mathrm{~cm}$ oberhalb der Fibulaspitze beginnt (Abb.6). Danach werden die Insertionen von LFC und LFTA dargestellt. 


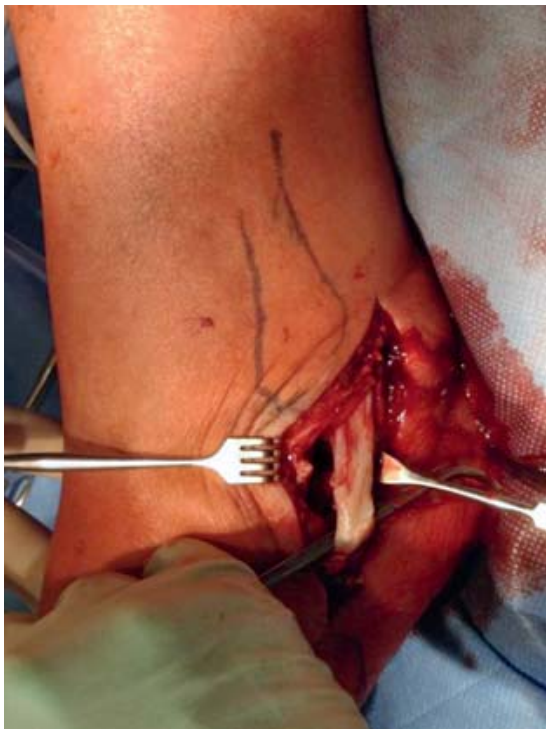

Abb. 5 Intraoperativer Situs bei Rekonstruktion der Peroneus-brevis-Sehne.

Die gemeinsame Insertion von LFC und LFTA befindet sich ca. $10-15 \mathrm{~mm}$ oberhalb der Fibulaspitze am anterioren Rand des Außenknöchels. Das LFTA verläuft von da aus horizontal und inseriert hinter der Knorpel-Knochen-Grenze ca. $17 \mathrm{~mm}$ proximal des Apex processus lateralis tali. Das LFC verläuft schräg nach distal und posterior. Zum Aufsuchen der LFC-Insertion am Kalkaneus eignet sich der Bildverstärker (ca. $15 \mathrm{~mm}$ unterhalb der proximalsten Spitze des Kalkaneus). Im Bereich der Insertionen werden dann Bohrlöcher angefertigt und das Sehnentransplantat eingezogen. Zur Fixation eignen sich entweder Interferenzschrauben mit kleinem Durchmesser oder Kippanker.

\section{Postoperative Behandlung}

Postoperativ wird der Fuß in einer funktionellen OSG-Orthese (z.B. Malleotristep) für 6 Wochen phasenadaptiert rehabilitiert. In dieser Zeit wird mit $20 \mathrm{~kg}$ Teilkörpergewicht belastet.

\section{Ersatzplastik der Syndesmose bei chronischer Instabilität}

Die Syndesmose besteht aus einem vorderen fibulotibialen Band (LTFA), einem hinteren fibulotibialen Band (LTFP) und einem interossären Band. Sie sichert die Fibula in ihrer Inzisur an der Tibia.

Chronische Instabilitäten der Syndesmose zwischen distaler Fibula und Tibia können zu deutlichen funktionellen Beeinträchtigungen führen. In diesen Fäl-

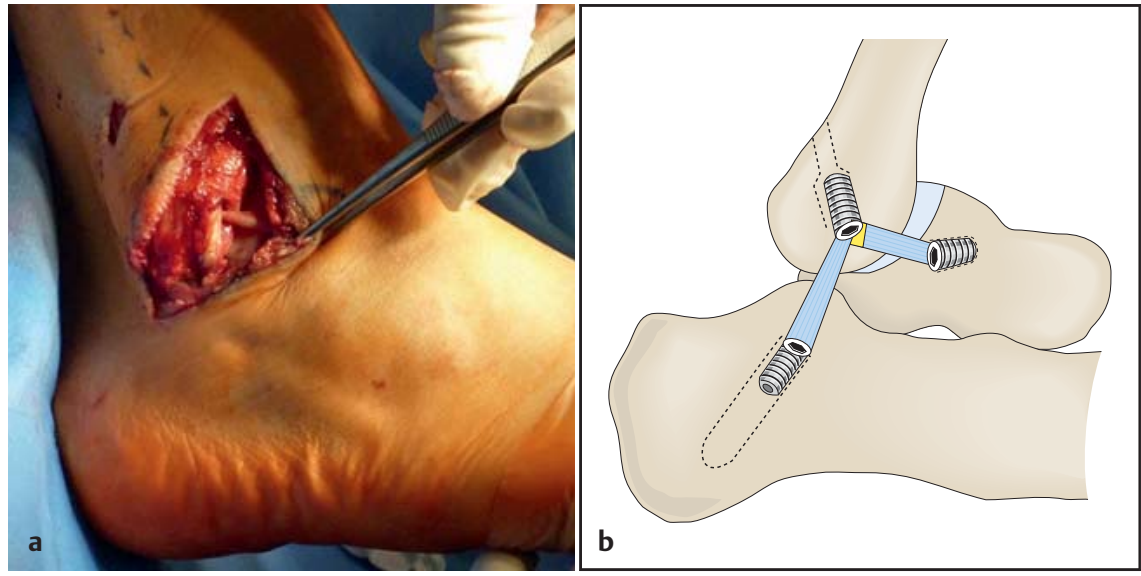

Abb. 6 a und $\mathbf{b}$ Rekonstruktion des lateralen Bandapparats des OSG. a Die gemeinsame Insertion von LFC und LFTA befindet sich ca. 10-15 mm oberhalb der Fibulaspitze am anterioren Rand des Außenknöchels. Das LFTA inseriert hinter der Knorpel-Knochen-Grenze am Talus ca. 17 mm proximal des Apex processus lateralis tali. Das LFC verläuft schräg nach distal und posterior. b Die Fixation erfolgt mit Interferenzschrauben.

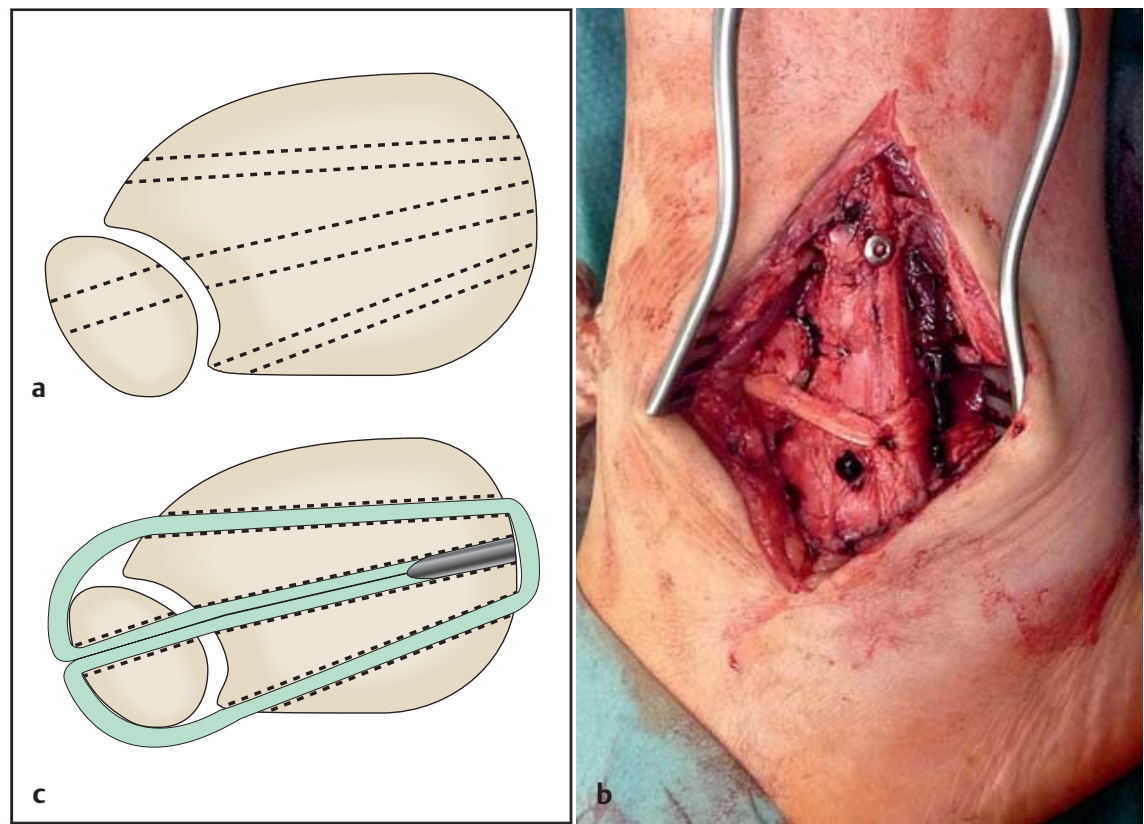

Abb. 7 a bis c Rekonstruktion der Syndesmose des OSG. a Es werden Bohrlöcher im Bereich der Insertionen von LTFP und LTFA in die Tibia gebohrt. Ein 3. Bohrloch führt durch Fibula und Tibia. b Das Sehnentransplantat wird mit einem Ende durch den anterioren Kanal und mit dem anderen Ende durch den posterioren Kanal gezogen. c Die Transplantatenden werden gemeinsam durch den mittleren Tunnel wieder nach medial ausgeleitet und gespannt. Zur Fixation eignet sich eine Interferenzschraube (Durchmesser ca. $6 \mathrm{~mm}$ ). Die Bandplastik wird anschließend mit einer Stellschraube gesichert, die ca. 2-3 cm proximal der Knochentunnel eingebracht wird.

len, kann eine Bandplastik indiziert sein. Als Transplantat für den anatomischen Ersatz aller 3 Bänder eignet sich die Semitendinosussehne $[3,4]$.

\section{OP-Technik}

Die Exposition der Syndesmose des OSG erfolgt über eine bogenförmige Inzision entlang der Außenknöchelvorderkante, die ca. $5 \mathrm{~cm}$ oberhalb der Fibulaspitze beginnt (Abb. 7).

Dann werden im Bereich der Insertionen von LTFP und LTFA Bohrlöcher in die Tibia gebohrt, die bis über die mediale Kortikalis reichen. Ein 3. Bohrloch wird knapp oberhalb des Gelenkspalts durch Fibula und Tibia gebohrt. Dann wird das Sehnentransplantat mit einem Ende durch den anterioren Kanal und mit 
dem anderen Ende durch den posterioren Kanal gezogen. Die Transplantatenden werden gemeinsam durch den mittleren Tunnel wieder nach medial ausgeleitet und gespannt. Zur Fixation eignet sich eine Interferenzschraube (Durchmesser ca. $6 \mathrm{~mm}$ ). Die Bandplastik wird anschließend mit einer Stellschraube gesichert, die ca. $2-3 \mathrm{~cm}$ proximal der Knochentunnel eingebracht wird.

\section{Postoperative Behandlung}

Postoperativ wird der Fuß in einer festen OSG-Orthese (z.B. Walker, Vacoped) für 6 Wochen immobilisiert. In dieser Zeit wird mit $20 \mathrm{~kg}$ Teilkörpergewicht belastet.

\section{Zusammenfassung}

Zusammenfassend eignen sich die Semitendinosus- und die Gracilissehne gut als autologe Transplantate zum Ersatz großer Sehnen und Bänder an der unteren Extremität.

\section{Literatur}

1 Dierckman BD, Ferkel RD. Anatomic reconstruction with a semitendinosus allograft for chronic lateral ankle instability. Am J Sports Med 2015; 43: 1941-1950

2 Dumbre Patil SS, Dumbre Patil VS, Basa VR et al. Semitendinosus tendon autograft for reconstruction of large defects in chronic achilles tendon ruptures. Foot Ankle Int 2014; 35: 699-705

${ }^{3}$ Lui TH. Tri-ligamentous reconstruction of the distal tibiofibular syndesmosis: a minimally invasive approach. J Foot Ankle Surg 2010; 49: 495-500

${ }^{4}$ Morris MW, Rice P, Schneider TE. Distal tibiofibular syndesmosis reconstruction using a free hamstring autograft. Foot Ankle Int 2009; 30: 506-511

5 Nguene-Nyemb AG, Huten $D$, Ropars $M$. Chronic patellar tendon rupture reconstruction with a semitendinosus autograft. Orthop Traumatol Surg Res 2011; 97: 447-450

${ }^{6}$ Petersen W, Zantop T. [Arthroscopic reconstruction of the anterolateral bundle of the posterior cruciate ligament in single-bundle technique with autologous hamstring grafts]. Oper Orthop Traumatol 2010; 22: 354-372

7 Petersen W, Forkel P, Achtnich A et al. [Anatomic reconstruction of the anterior cruciate ligament in single bundle technique]. Oper Orthop Traumatol 2013; 25: 185-204
${ }^{8}$ Squires N, Myerson MS, Gamba C. Surgical treatment of peroneal tendon tears. Foot Ankle Clin 2007; 12: 675-695

9 Takazawa Y, Ikeda H, Ishijima $\mathrm{M}$ et al. Reconstruction of a ruptured patellar tendon using ipsilateral semitendinosus and gracilis tendons with preserved distal insertions: two case reports. BMC Res Notes 2013; 6: 361

Prof. Dr. med. W. Petersen

Chefarzt

Dr. med. Ute Petersen

Fachärztin

Dr. med. Cara Winter

Oberärztin

Klinik für Orthopädie und

Unfallchirurgie

Martin-Luther-Krankenhaus, Berlin,

Grunewald

Caspar-Theyß-Straße 27-31

14193 Berlin

wolf.petersen@pgdiakonie.de 